\title{
Extended Kalman Filter-Based Approach for Autonomous Synchronization and Ranging in GPS-Denied Environments
}

\author{
Xiaobo Gu $\mathbb{D}^{1},{ }^{1,2}$ Weiqiang Tan $\left(\mathbb{D},{ }^{3}\right.$ Di Zhang $\mathbb{D}^{4},{ }^{4}$ Yudong Lu $\mathbb{D}^{5},{ }^{5}$ and Ruidian Zhan $\mathbb{i}^{6}$ \\ ${ }^{1}$ School of Automation, Guangdong University of Technology, Guangzhou 510000, China \\ ${ }^{2}$ Research Institute of Integrated Circuit Innovation, Guangdong University of Technology, Guangzhou 510006, China \\ ${ }^{3}$ School of Computer Science and Cyber Engineering, Guangzhou University, Guangzhou 510006, China \\ ${ }^{4}$ The Second Military Agent Office, Equipment Department of China PLA Air Force in Beijing Area, Beijing 100074, China \\ ${ }^{5}$ Guangzhou GRG Metrology \& Test Co., Ltd., Guangzhou 510656, China \\ ${ }^{6}$ Chipeye Microelectronics Foshan Ltd., Foshan 528225, China
}

Correspondence should be addressed to Ruidian Zhan; zhanruidian@chipeye.cn

Received 25 August 2020; Revised 14 November 2020; Accepted 6 December 2020; Published 19 December 2020

Academic Editor: Xin Dong

Copyright (C) 2020 Xiaobo Gu et al. This is an open access article distributed under the Creative Commons Attribution License, which permits unrestricted use, distribution, and reproduction in any medium, provided the original work is properly cited.

Network ranging and clock synchronization based on two-way timing stamps exchange mechanism in complex GPS-denied environments is addressed in this paper. An estimator based on the Extended Kalman filter (EKF) is derived, according to which, the clock skew, clock offset, and ranging information can be jointly estimated. The proposed estimator provides off-line computation by storing the transmitting timing stamps in advance and could be implemented in asymmetrical and asynchronous scenarios. The simulation results show that the proposed estimator achieves a relative good performance than the existed estimators. In addition, a new Bayesian Cramér-Rao Lower Bound (B-CRLB) is derived. Numerous simulation results show that the proposed estimator meets the B-CRLB.

\section{Introduction}

The Unmanned Autonomous System (UAS) [1-3] has become a hotspot in the past decades with the development of technology. In order to carry out unmanned and autonomous missions, the real-time information of positions, velocities, and timing tags of the system agents are required to be collected. A common approach is to deploy Global Positioning System (GPS) receivers on the network members, which is able to provide precise and accurate Positioning, Velocity, and Timing (PVT) service [4]. However, such a design is no longer available in the complex scenarios where GPS service is not sky visible or of low quality: underwater, valley, indoors, and underground. To solve this problem, the fixed anchor nodes with known positions are implemented to substitute for the PVT service of GPS [5-8]. By broadcasting its timing information, the network agents in the certain area could be able to perform relative positioning, motion estimation and cooperative missions by way of Time of Arrival (TOA) [9] and Time Difference of Arrival (TDOA) [10] in complex environments. It is noted that achieving time synchronization among the anchor nodes is the first step to carry out the subsequence missions.

Normally, the anchor nodes are of low overall cost, which implies that the on-board oscillators are not highly qualified and the measurement devices are not absolutely accurate. Moreover, the nodes might be affected by the external environmental changes as well. These factors bring a problem that the impacts owing to the relative clock skews and offsets between the clocks could not be ignored [11]. How to correct these errors becomes paramount, especially for the UASs which require all the anchor nodes to provide the synchronized timing tags. Furthermore, power consumption is another important issue for the low-end sensor nodes. Therefore, the message transaction times among the anchor nodes and the message itself should be as few as possible.

In order to estimate both clock skew and clock offset under unknown delay, a Maximum Likelihood-like 
Estimator (MLLE) was derived in [12]. A step further, Leng and $\mathrm{Wu}$ proposed a Low Complexity Least Square (LCLS) [13] method which outperforms MLLE. Rajan and van der Veen proposed a Global Least Square (GLS) method [14], which was an extension of the LCLS estimator and had the function of jointly estimating the clock offsets, clock skews, and relative distances in an anchor network or even in an anchorless network [15]. An algorithm based on the alternating direction method of multipliers was proposed in [16], and the simulations results show that it outperforms the distributed least squares algorithm. In addition, Luo and $\mathrm{Wu}$ proposed an approach based on Kalman Filter (KF) [17], according to which, the accumulated clock offsets and clock skews were estimated once each pair of forward link and corresponding reverse link was collected. A similar scheme was also proposed in [18], which extended the state estimation model to a clock adjustment approach.

To the best of our knowledge, the existed estimators based on two-way timing stamp exchange mechanism and the KF require both the information of the forward and reverse links to difference away the time of flight when performing estimation. This kind of property leads to two problems: one is that there might be a long idle time especially when the time interval between each round transaction is very long and the other problem is that this type of structure could not deal with the circumstance of transaction link loss.

The main contribution of this paper is to propose a novel estimator in the context of complex environments, by which the unknown clock skew, clock offset, and propagation delay can be jointly estimated. The proposed estimator adopts EKF to iteratively update the state estimation on clock parameters and ranging measurements. The two-way timing stamp exchange mechanism is implemented as a basic element to establish the communication network. Moreover, for the sake of decreasing the burden on memory, the transmitting time of each node are recorded in advance so that the EKF estimator can provide explicit off-line computation of the estimation error confidence intervals, since these do not depend on the real-time measurements. Unlike the LS-based estimators, which are less than desirable to collect sufficient observations before doing an update in one big calculation, the proposed estimator could perform the updates once a new observation is collected.

Notation: $(\cdot)_{k}^{+}$stands for $(\cdot)$ at time index $k+1, \operatorname{diag}(\cdot)$ denotes a diagonal matrix formed from its vector argument, the transposition is denoted by $(\cdot)^{T}$, the matrices are denoted by boldface letters, and $\mathbf{I}_{N}$ denotes an identity matrix of size N.

\section{System Model}

We consider a fully asynchronous network consisting of $N$ anchor nodes. Each anchor node deploys an imperfect frequency source, and the clock skews and the clock offsets among the network agents are not identical due to various reasons. The relative distance between each pair of anchor nodes is fixed and unknown.
2.1. Clock Model. Since the free-running clock is not ideal owing to various external and internal reasons, the clock error must be taken into consideration. Let $t$ be the global time and $t_{i}$ be the local time of node $i$, then the relationship between $t$ and $t_{i}$ could be expressed by

$$
t_{i}=\omega_{i} t+\phi_{i} \Leftrightarrow C_{i}\left(t_{i}\right) \triangleq t=\alpha_{i} t_{i}+\beta_{i},
$$

where $\omega_{i} \in \mathbb{R}_{+}$and $\phi_{i} \in \mathbb{R}$ denote the clock skew and the initial clock offset of node $i$, respectively, $C_{i}\left(t_{i}\right)$ denotes the global time of node $i$ at local time $t_{i}$, and $\alpha_{i} \in \mathbb{R}_{+}$and $\beta_{i} \in \mathbb{R}$ are virtual parameters derived from $\omega_{i}$ and $\phi_{i}$. For an autonomous time synchronization system, generally, a real or virtual clock is elected as the reference. Let node 1 be the reference in this paper, that is to say, $\omega_{1}=1$ and $\phi_{1}=0$, which is equivalent to $\alpha_{1}=1$ and $\beta_{1}=0$. Comparing the two equations in (1) and extending to a vector form yields

$$
\begin{aligned}
& \boldsymbol{\alpha}=1_{N-1} \oslash \boldsymbol{\omega}, \\
& \boldsymbol{\beta}=-\phi \oslash \boldsymbol{\omega},
\end{aligned}
$$

where $\omega=\left[\omega_{2}, \ldots, \omega_{N}\right]^{T} \in \mathbb{R}_{+}^{(N-1) \times 1}, \phi=\left[\phi_{2}, \ldots, \phi_{N}\right]^{T} \in$ $\mathbb{R}^{(N-1) \times 1}, \alpha=\left[\alpha_{2}, \ldots, \alpha_{N}\right]^{T} \in \mathbb{R}_{+}^{(N-1) \times 1}$, and $\beta=\left[\beta_{2}, \ldots\right.$, $\left.\beta_{N}\right]^{T} \in \mathbb{R}^{(N-1) \times 1}$.

It is worth noting that the clock skew might vary versus time and the clock drift term might not be ignored in some cases. However, the proposed model could be reasonable for a small period of time and has been largely adopted in the network clock synchronization literature.

2.2. Ranging Model. The two-way timing stamp exchange mechanism with a centralized scenario, as shown in Figure 1, is implemented to realize time synchronization and ranging in this paper. The two nodes are capable of communicating with each other with a master-slave structure. For the $k$ th forward link between node $i$ and node $j$, two timing stamps $\left\{T_{1, i j}^{k}, T_{2, i j}^{k}\right\}$ are collected by this round of communication, which represent the transmitting time instant and reception time instant, respectively. Similarly, the timing stamps $\left\{T_{3, i j}^{k}, T_{4, i j}^{k}\right\}$ could be collected by the reverse link as well. All the data of two-way communication timing stamps are stored in the center processing unit to preform estimation. The $k$ th forward link and reverse link can be modelled as

$$
\begin{aligned}
& C_{j}\left(T_{2, i j}^{k}+n_{1}^{k}\right)-C_{i}\left(T_{1, i j}^{k}+n_{2}^{k}\right)=\tau_{i, j}+n_{3}^{k}, \\
& C_{i}\left(T_{4, i j}^{k}+n_{4}^{k}\right)-C_{j}\left(T_{3, i j}^{k}+n_{5}^{k}\right)=\tau_{j, i}+n_{6}^{k},
\end{aligned}
$$

where $\left.\left\{n_{1}^{k}, n_{2}^{k}, n_{4}^{k}, n_{5}^{k}\right\} \sim \mathrm{N}\left(0, \sigma_{p}^{2}\right)\right\}$ and $\left\{n_{3}^{k}, n_{6}^{k}\right\} \sim \mathrm{N}\left(0, \sigma_{q}^{2}\right)$ are independent aggregate Gaussian noise variables, which originate from measurements and space disturbances, respectively. $\tau_{i, j}$ denotes the propagation delay between node $i$ and node $j$. Since the relative positions of the anchor nodes are fixed, the propagation delays of the forward links and reverse links for each pair of master and slave nodes are identical, namely, $\tau_{i, j}=\tau_{j, i}$. It can be seen that the maximum number of direct communication links is $L=(N ! /(N-2) !)$ when there is a full connection topology. The unknown propagation delay is expressed as $\tau=\left[\tau_{1,2}, \ldots, \tau_{1, N}\right.$, 


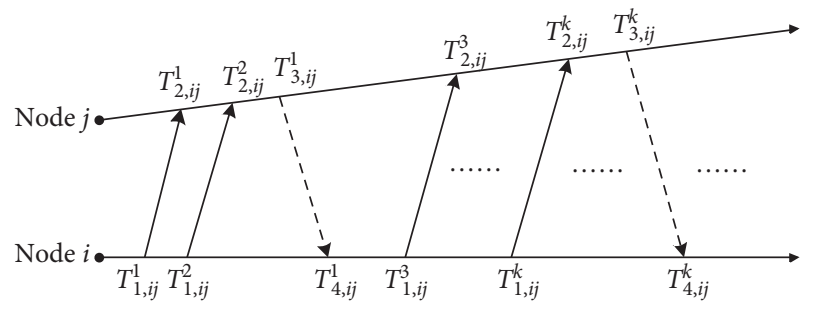

FIgURE 1: Two-way timing stamp exchange mechanism between node $i$ and node $j$. The solid (dashed) lines denote the forward (reverse) links.

$\left.\tau_{2,3}, \ldots, \tau_{2, N}, \tau_{(N-1), N},\right]^{T} \in \mathbb{R}^{(L / 2) \times 1}$. Substituting (1) into (3) and (4) yields

$$
\begin{aligned}
& \alpha_{i} T_{1, i j}^{k}=\alpha_{j} T_{2, i j}^{k}-\beta_{i}+\beta_{j}-\tau_{i, j}+\alpha_{j} n_{1}^{k}-\alpha_{i} n_{2}^{k}-n_{3}^{k}, \\
& \alpha_{i} T_{4, i j}^{k}=\alpha_{j} T_{3, i j}^{k}-\beta_{i}+\beta_{j}+\tau_{i, j}-\alpha_{i} n_{4}^{k}+\alpha_{j} n_{5}^{k}+n_{6}^{k} .
\end{aligned}
$$

Rearranging (5) and (6) yields

$$
\begin{aligned}
& T_{2, i j}^{k}=\frac{\alpha_{i} T_{1, i j}^{k}}{\alpha_{j}}+\frac{\beta_{i}}{\alpha_{j}}-\frac{\beta_{j}}{\alpha_{j}}+\frac{\tau_{i, j}}{\alpha_{j}}-n_{1}^{k}+\frac{\alpha_{i} n_{2}^{k}}{\alpha_{j}}+\frac{n_{3}^{k}}{\alpha_{j}}, \\
& T_{4, i j}^{k}=\frac{\alpha_{j} T_{3, i j}^{k}}{\alpha_{i}}-\frac{\beta_{i}}{\alpha_{i}}+\frac{\beta_{j}}{\alpha_{i}}+\frac{\tau_{i, j}}{\alpha_{i}}-n_{4}^{k}+\frac{\alpha_{j} n_{5}^{k}}{\alpha_{i}}+\frac{n_{6}^{k}}{\alpha_{i}} .
\end{aligned}
$$

2.3. Proposed Estimator. By combining the clock model and ranging model, a new state estimation model is proposed in this paper. According to this model, the clock error and ranging error could be jointly considered and estimated by the discrete state estimation method.

Substituting (2) into (5) and (6) leads to

$$
\begin{aligned}
& T_{2, i j}^{k}=\frac{T_{1, i j}^{k} \omega_{j}}{\omega_{i}}-\frac{\phi_{i} \omega_{j}}{\omega_{i}}+\phi_{j}+\tau_{i, j} \omega_{j}-n_{1}^{k}+\frac{n_{2}^{k} \omega_{j}}{\omega_{i}}+n_{3}^{k} \omega_{j}, \\
& T_{4, i j}^{k}=\frac{T_{3, i j}^{k} \omega_{i}}{\omega_{j}}-\frac{\phi_{j} \omega_{i}}{\omega_{j}}+\phi_{i}+\tau_{i, j} \omega_{i}-n_{4}^{k}+\frac{n_{5}^{k} \omega_{i}}{\omega_{j}}+n_{6}^{k} \omega_{i} .
\end{aligned}
$$

It is worth noting that the transmitting time series $T_{1, i j}^{k}$ and $T_{3, i j}^{k}$ are timing tags recorded by the member nodes. For the sake of convenience and building up the discrete time state model, they normally are set as periodic series. In this case, $T_{1, i j}^{k}$ and $T_{3, i j}^{k}$ could be considered as known parameters, and the left sides of (7) and (8) could be considered as observations. The state estimation could be updated once a new observation has been collected. Therefore, this process can be expressed by a nonlinear state estimation model:

$$
\left\{\begin{array}{l}
\mathbf{X}_{k}^{+}=\mathbf{A X}_{k}+\mathbf{r}_{k}, \\
\mathbf{z}_{k}=\mathbf{f}_{k}\left(\mathbf{X}_{k}\right)+\mathbf{v}_{k},
\end{array}\right.
$$

where the unknown state matrix $\mathbf{X}_{k}=[\omega, \phi, \tau]^{T} \epsilon$ $\mathbb{R}^{(2 N-2+(L / 2)) \times 1} \cdot \mathbf{A}=\mathbf{I}_{(2 N-2+(L / 2))}$ and $\left\{\mathbf{v}_{k}\right\}$ are white Gaussian, independent random processes with zero mean and covariance matrix $E\left[\mathbf{r}_{k} \mathbf{r}_{k}^{T}\right]=\mathbf{R}_{k} \in \mathbb{R}^{(2 N-2+(L / 2)) \times(2 N-2+(L / 2))}$ and
$E\left[\mathbf{v}_{k} \mathbf{v}_{k}^{T}\right]=\mathbf{Q}_{k} \in \mathbb{R}^{1 \times 1} . \mathbf{f}_{k}\left(\mathbf{X}_{k}\right)$ varies versus the direction of the link. From (9) and (10), we can infer that if the transmission direction is from node $i$ to node $j$, the observation equation follows (9), otherwise it follows (10). The estimation could be performed by an extended Kalman filter (EKF) in this case. In addition, if the two-way timing stamps exchange mechanism operates between the master and slave, the observation function $\mathbf{f}_{k}\left(\mathbf{X}_{k}\right)$ can be simplified as $T_{1, i j}^{k} \omega_{j}+\phi_{j}+$ $\tau_{i, j} \omega_{j}$ and $\left(T_{3, i j}^{k} / \omega_{j}\right)-\left(\phi_{j} / \omega_{j}\right)+\tau_{i, j}$, respectively.

Based on the discussions above, the proposed EKF estimator is expressed by Algorithm 1.

Remark 1 (off-line computation). Note that the matrices $\mathbf{K}$ and $\mathbf{P}_{m \mid m}$ can be computed off-line; the reason is that they do not depend on the timing stamp data of reception. Therefore, the computation of $\widehat{X}$ can be performed very rapidly on the center processing unit. It can be found that one disadvantage of EKF is that it might take a large amount of memories to store the transmission data especially when the data is large, but this problem could be easily solved by setting the transmission time instants periodically.

Remark 2 (real-time process). Compared with the existed LS-based estimators, which need to collect the information with respect to forward links and reverse links as much as possible to improve the estimation accuracy, EKF could update its estimation once there is a new observation of a forward or a reverse link. On the contrary, although the Kalman-filter-based algorithms proposed in $[17,18]$ are also able to carry out real-time processing, the symmetrical links are required to eliminate the nuisance term. In addition, another advantage owing to not to put any constraint on symmetrical structure is that the update rate could be faster than those symmetrical required algorithms. This kind of feature is paramount especially for the low power consumption systems, such as the sleep wake-up scheduling system.

Remark 3 (one-way estimation). According to (7) and (8), we can infer that the EKF estimator will not lose its entire function even when the transmitting device or receiving device on any node suddenly stops working. For instance, if (10) could not be observed, that is to say, only a series of information with respect to (9) can be used to perform estimation, in this case, the estimation rate and the robustness of the estimator could be improved.

\section{Bayesian Cramér-Rao Lower Bound}

The Cramér-Rao lower bound on the estimation error states

$$
E\left[(\widehat{X}-\mathbf{X})(\widehat{X}-\mathbf{X})^{T}\right] \geq \mathbf{J}^{-1},
$$

where $\mathbf{J}$ is the Fisher information matrix, which obeys the recursion [19]:

$$
\mathbf{J}_{k+1}=\mathbf{D}_{k}^{22}-\left(\mathbf{D}_{k}^{12}\right)^{T}\left(\mathbf{J}_{k}+\mathbf{D}_{k}^{11}\right)^{-1} \mathbf{D}_{k}^{12}
$$

where 


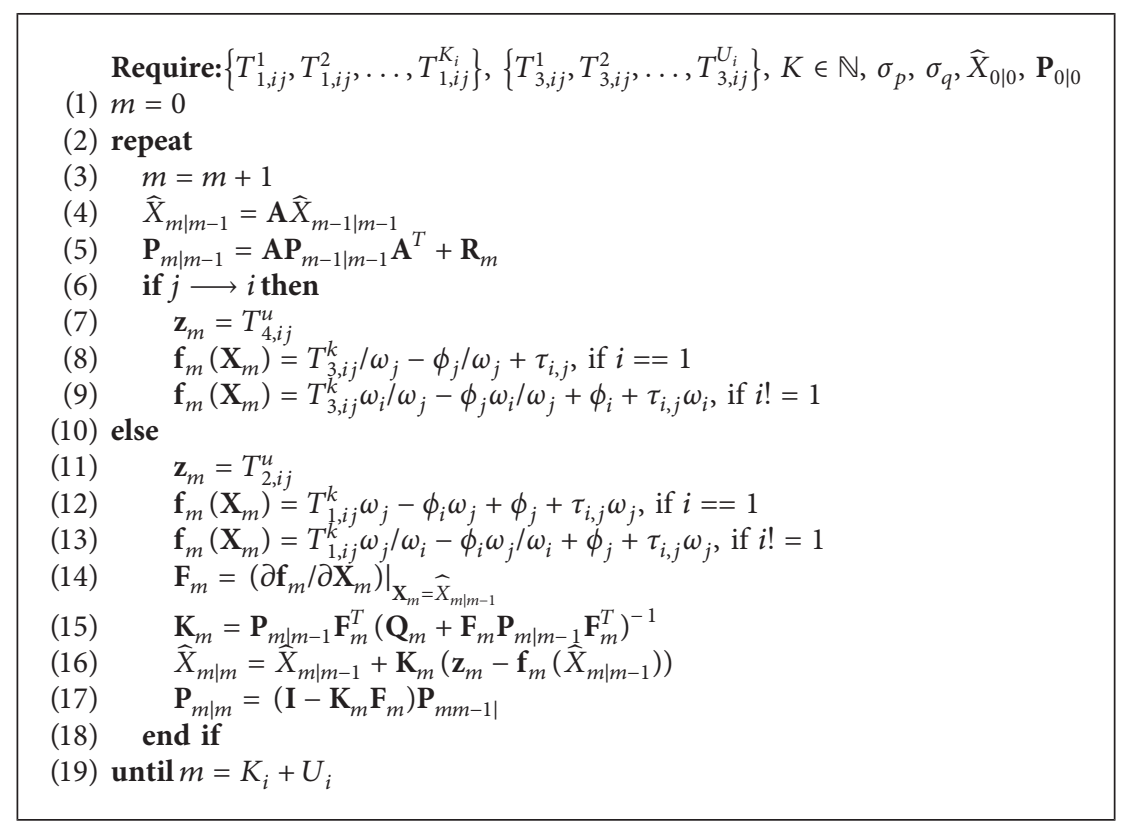

Algorithm 1: Proposed EKF estimator.

$$
\begin{aligned}
\mathbf{D}_{k}^{11}= & E\left\{-\frac{\partial^{2}}{\partial \mathbf{X}_{k} \partial^{T} \mathbf{X}_{k}} \log p\left(\mathbf{X}_{k}^{+} \mid \mathbf{X}_{k}\right)\right\}, \\
\mathbf{D}_{k}^{12}= & E\left\{-\frac{\partial^{2}}{\partial \mathbf{X}_{k} \partial^{T} \mathbf{X}_{k}^{+}} \log p\left(\mathbf{X}_{k}^{+} \mid \mathbf{X}_{k}\right)\right\}, \\
\mathbf{D}_{k}^{22}= & E\left\{-\frac{\partial^{2}}{\partial \mathbf{X}_{k}^{+} \partial^{T} \mathbf{X}_{k}^{+}} \log p\left(\mathbf{X}_{k}^{+} \mid \mathbf{X}_{k}\right)\right\} \\
& +E\left\{-\frac{\partial^{2}}{\partial \mathbf{X}_{k}^{+} \partial^{T} \mathbf{X}_{k}^{+}} \log p\left(z_{k}^{+} \mid \mathbf{X}_{k}^{+}\right)\right\} .
\end{aligned}
$$

From (11), it follows that

$$
\begin{aligned}
& -\log p\left(\mathbf{X}_{k}^{+} \mid \mathbf{X}_{k}\right)=c_{1}+\frac{1}{2}\left(\mathbf{X}_{k}^{+}-\mathbf{A} \mathbf{X}_{k}\right)^{T} \cdot \mathbf{R}^{-1}\left(\mathbf{X}_{k}^{+}-\mathbf{A} \mathbf{X}_{k}\right) \\
& -\log p\left(z_{k}^{+} \mid \mathbf{X}_{k}^{+}\right)=c_{2}+\frac{1}{2}\left[z_{k}^{+}-\mathbf{f}_{k}^{+}\left(\hat{X}_{k}^{+}\right)\right]^{T} \cdot \mathbf{Q}^{-1}\left[z_{k}^{+}-\mathbf{f}_{k}^{+}\left(\hat{X}_{k}^{+}\right)\right]
\end{aligned}
$$

where $c_{1}$ and $c_{2}$ are constants. A straightforward calculation of (14)-(18) leads to $\mathbf{D}_{k}^{11}=\mathbf{A}^{T} \mathbf{R}^{-1} \mathbf{A}, \mathbf{D}_{k}^{12}=-\mathbf{A}^{T} \mathbf{R}^{-1}$, and $\mathbf{D}_{k}^{22}=\mathbf{R}^{-1}+\left\{\left(\partial \mathbf{f}_{k}^{+}\left(\widehat{X}_{k}^{+}\right) / \partial \widehat{X}_{k}^{+}\right)\right\}^{T} \mathbf{Q}^{-1}\left(\partial \mathbf{f}_{k}^{+}\left(\widehat{X}_{k}^{+}\right) / \partial \widehat{X}_{k}^{+}\right)$. Hence, (13) can be written as

$$
\begin{aligned}
\mathbf{J}_{k+1}= & \mathbf{R}^{-1}+\left\{\frac{\partial \mathbf{f}_{k}^{+}\left(\widehat{X}_{k}^{+}\right)}{\partial \widehat{X}_{k}^{+}}\right\}^{T} \mathbf{Q}^{-1} \frac{\partial \mathbf{f}_{k}^{+}\left(\widehat{X}_{k}^{+}\right)}{\partial \widehat{X}_{k}^{+}} \\
& -\left(\mathbf{R}^{-1}\right)^{T} \mathbf{A}\left(\mathbf{J}_{k}+\mathbf{A}^{T} \mathbf{R}^{-1} \mathbf{A}\right)^{-1} \mathbf{A}^{T} \mathbf{R}^{-1}
\end{aligned}
$$

Applying the matrix inversion lemma, (19) can be further simplified as

$$
\mathbf{J}_{k+1}=\left(\mathbf{R}+\mathbf{A J}_{k}^{-1} \mathbf{A}^{T}\right)^{-1}+\left\{\frac{\partial \mathbf{f}_{k}^{+}\left(\widehat{X}_{k}^{+}\right)}{\partial \widehat{X}_{k}^{+}}\right\}^{T} \mathbf{Q}^{-1} \frac{\partial \mathbf{f}_{k}^{+}\left(\widehat{X}_{k}^{+}\right)}{\partial \widehat{X}_{k}^{+}}
$$

The inverse of the Fisher information matrix for $\theta$ could be written as [20]

$$
\mathbf{I}^{-1}(\boldsymbol{\theta})=\mathbf{J}^{-1}
$$

\section{Simulations}

In this section, we provide a scenario with asynchronous links to evaluate the performance of the EKF estimator. The number of the nodes $N=10$, and each node is capable of performing two-way communications with the other nodes. All the member nodes are assumed to be visible during the signal transaction process. The locations of the member nodes are uniformly distributed variables in the region of $1 \mathrm{~km} \times 1 \mathrm{~km}$. The propagation speed is the speed of light. The clock skew and clock offset of the slave nodes are randomly distributed in the range $[0.9998,1.0002]$ and $[-2,2] s$, respectively. The transmission time interval for both forward links and reverse links is $5 \mathrm{~s}$, and the reverse links always transmit backwards by $3 \mathrm{~s}$. The noise standard deviations $\sigma_{p}=1 \times 10^{-3}$ and $\sigma_{q}=1 \times 10^{-3}$. The Root Mean Square Error (RMSE) is adopted as the performance metric. All the given results are averaged over 10,000 Monte Carlo runs.

In order to investigate the performance of the proposed method, the conventional Kalman estimator based on the traditional clock model and the iterative form of LCLS are compared in this section. Figure 2 shows the RMSEs of the unknown parameters by applying different estimators. As shown in the figure, the iterative LCLS achieve the best performance than the other estimators. The proposed EKF 

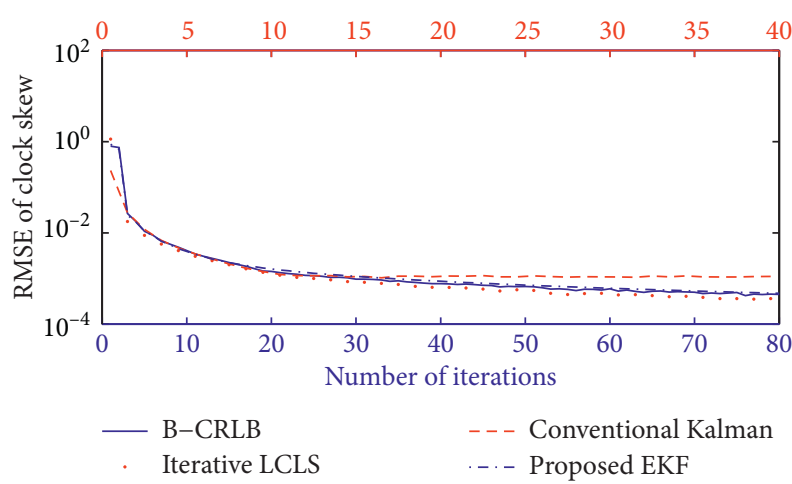

(a)

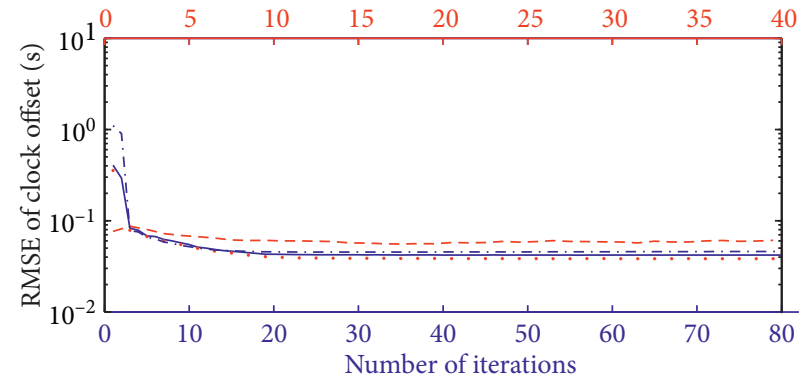

B-CRLB

Iterative LCLS
- - - Conventional Kalman . . - - Proposed EKF

(b)

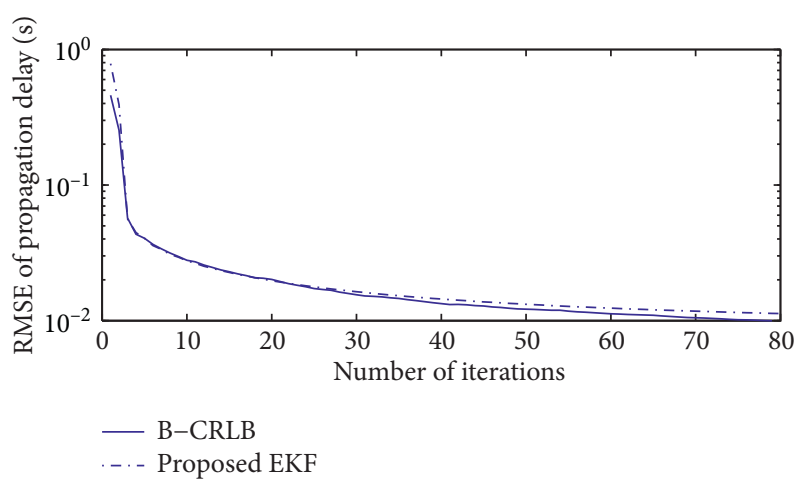

(c)

FIGURE 2: RMSEs of the estimated clock skew $\omega_{j}(\mathrm{a})$, offset $\phi_{j}(\mathrm{~b})$, and propagation delay $\tau_{i, j}(\mathrm{c})$ with respect to the number of iterations.

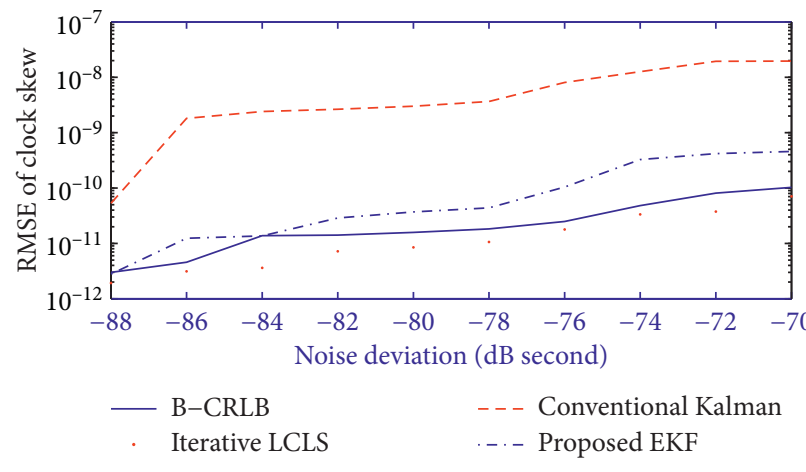

(a)

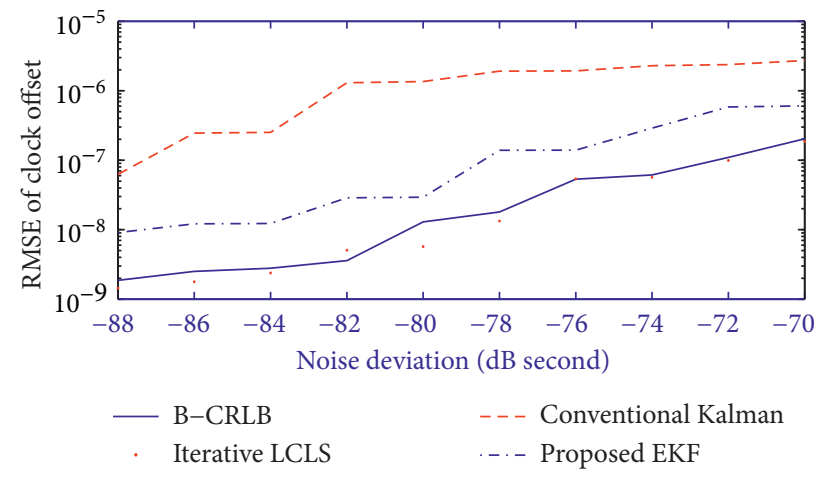

(b)

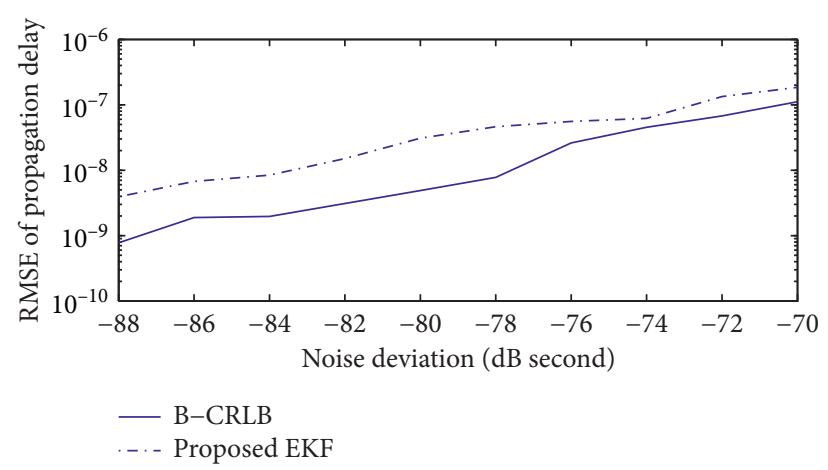

(c)

FIGURE 3: RMSEs of the estimated clock skew $\omega_{j}(\mathrm{a})$, offset $\phi_{j}(\mathrm{~b})$, and propagation delay $\tau_{i, j}(\mathrm{c})$ with respect to the varying noise deviations. 
converges at a slower rate than the conventional $\mathrm{KF}$, but performs relatively better on the estimation precision after a long iteration time. Compared with the other two estimators, the precision degradation of the EKF originates from the nonlinear process of the observation equation, and it could be improved by omitting a virtual parameter transforming process. However, the proposed EKF estimator has its own superiorities mentioned in Section 2, namely, its update rate is two times faster than the other estimators and it frees the restriction on the symmetrical structure. Meanwhile, it is able to provide off-line computation.

Furthermore, the performances of the estimators for varying noise deviation are presented in Figure 3. The deviation of the time markers and propagation disturbance are set in the range $[-88,-70] \mathrm{dB}$ seconds. It is noted that the meter level accuracies could be achieved by the proposed EKF estimator when the Signal-to-Noise Ratio (SNR) is high. In addition, the proposed estimator is able to carry out range estimation directly, which is coupled with the estimation process of clock parameter tracking.

\section{Conclusion}

In this work, the problem of joint clock synchronization and ranging for UAS in a complex GPS-denied environment is addressed. The proposed EKF estimator is shown to achieve nearly the same performance compared with the existed Kalman-filter-based estimator, but can provide off-line computations. By comparing its performance bounds with the B-CRLB, it has been found that although the EKF estimator suffers performance degradation, it is capable of estimating the clock skew even in the context of one-way communication and updating the estimates faster than the existed estimators. Furthermore, it has the function of estimating the unknown propagation delay between each node. Future research directions include the extension of recursive joint clock synchronization and localization estimation in anchorless networks.

\section{Data Availability}

The data used to support the findings of this study are available from the corresponding author upon reasonable request.

\section{Conflicts of Interest}

The authors declare that they have no conflicts of interest.

\section{Acknowledgments}

This work was supported by the Key-Area Research \& Development Program of Guangdong Province under Grant 2019B010142001, and the Science \& Technology Program of Guangdong under Grant 2019A141401005 and the National Natural Science Foundation of China under Grant 61801132.

\section{References}

[1] A. Zolich, D. Palma, K. Kansanen et al., "Survey on communication and networks for autonomous marine systems," Journal of Intelligent \& Robotic Systems, vol. 95, no. 3-4, pp. 789-813, 2019.

[2] Q. P. Ha, L. Yen, and C. Balaguer, "Robotic autonomous systems for earthmoving in military applications," Automation in Construction, vol. 107, p. 102934, 2019.

[3] R. Yang, A. Zhang, L. Zhang, and Y. Hu, "A novel adaptive h-infinity cubature kalman filter algorithm based on sagehusa estimator for unmanned underwater vehicle," Mathematical Problems in Engineering, vol. 2020, Article ID 8057028, 10 pages, 2020.

[4] E. Kaplan and C. Hegarty, Understanding GPS: Principles and Applications, Artech House, Norwood, MA, USA, 2005.

[5] Y. Xiong, N. Wu, Y. Shen, and M. Z. Win, "Cooperative network synchronization: asymptotic analysis," IEEE Transactions on Signal Processing, vol. 66, no. 3, pp. 757-772, 2017.

[6] J. Liu, Z. Wang, J.-H. Cui, S. Zhou, and B. Yang, "A joint time synchronization and localization design for mobile underwater sensor networks," IEEE Transactions on Mobile Computing, vol. 15, no. 3, pp. 530-543, 2015.

[7] N. M. Freris, S. R. Graham, and P. Kumar, "Fundamental limits on synchronizing clocks over networks," IEEE Transactions on Automatic Control, vol. 56, no. 6, pp. 1352-1364, 2010.

[8] R. M. Vaghefi and R. M. Buehrer, "Cooperative joint synchronization and localization in wireless sensor networks," IEEE Transactions on Signal Processing, vol. 63, no. 14, pp. 3615-3627, 2015.

[9] K. Yu and I. Oppermann, "Performance of uwb position estimation based on time-of-arrival measurements," in Proceedings of the 2004 International Workshop on Ultra Wideband Systems Joint with Conference on Ultra Wideband Systems and Technologies, pp. 400-404, IEEE, Kyoto, Japan, May 2004.

[10] L. Cong and W. Zhuang, "Non-line-of-sight error mitigation in tdoa mobile location," in Proceedings of the GLOBECOM'01, IEEE Global Telecommunications Conference, pp. 680-684, IEEE, San Antonio, TX, USA, November 2001.

[11] Y. S. Shmaliy, S. H. Khan, S. Zhao, and O. Ibarra-Manzano, "General unbiased fir filter with applications to gps-based steering of oscillator frequency," IEEE Transactions on Control Systems Technology, vol. 25, no. 3, pp. 1141-1148, 2016.

[12] K.-L. Noh, Q. M. Chaudhari, E. Serpedin, and B. W. Suter, "Novel clock phase offset and skew estimation using two-way timing message exchanges for wireless sensor networks," IEEE Transactions on Communications, vol. 55, no. 4, pp. 766-777, 2007.

[13] M. Leng and Y.-C. Wu, "On clock synchronization algorithms for wireless sensor networks under unknown delay, Vehicular Technology," IEEE Transactions on, vol. 59, no. 1, pp. 182-190, 2010.

[14] R. T. Rajan and A.-J. Van der Veen, "Joint ranging and clock synchronization for a wireless network," in Proceedings of the 2011 4th IEEE International Workshop on Computational Advances in Multi-Sensor Adaptive Processing (CAMSAP), pp. 297-300, IEEE, San Juan, Puerto Rico, December 2011.

[15] R. T. Rajan, G. Leus, and A.-J. van der Veen, "Relative kinematics of an anchorless network," Signal Processing, vol. 157, pp. 266-279, 2019. 
[16] B. Luo, L. Cheng, and Y.-C. Wu, "Fully distributed clock synchronization in wireless sensor networks under exponential delays," Signal Processing, vol. 125, pp. 261-273, 2016.

[17] B. Luo and Y. C. Wu, "Distributed clock parameters tracking in wireless sensor network," IEEE Transactions on Wireless Communications, vol. 12, no. 12, pp. 6464-6475, 2013.

[18] G. Giorgi and C. Narduzzi, "Performance analysis of kalmanfilter-based clock synchronization in IEEE 1588 networks," IEEE Transactions on Instrumentation and Measurement, vol. 60, no. 8, pp. 2902-2909, 2011.

[19] P. Tichavskỳ, C. H. Muravchik, and A. Nehorai, "Posterior cramér-rao bounds for discrete-time nonlinear filtering," IEEE Transactions on Signal Processing, vol. 46, no. 5, pp. 1386-1396, 1998.

[20] S. M. Kay, Fundamentals of Statistical Signal Processing: Practical Algorithm Development, Pearson Education, London, UK, 2013. 